\title{
Sepsis: What We Know and What We Don't
}

\section{Jyotika sharma*}

Department of Microbiology and Immunology, UND School of Medicine and Health Sciences, 501 N Columbia Road, Grand Forks, ND 58202-9037, USA

Sepsis is a complex immune disorder that results in 750,000 hospitalizations annually in the United States alone [1]. In addition to posing an immense health hazard with a mortality rate of $20-50 \%$, it is also a huge economic healthcare burden with the patients consuming half of the ICU resources in the country. In October 2011, the only FDA approved drug for the treatment of sepsis (Xigris) was withdrawn from market following the much anticipated results of the clinical trial (PROWESS-SHOCK) where it failed to show any survival benefit for severe sepsis and septic shock patients [2]. As a result we are now left with no effective preventive or treatment options for this deadly immune disorder, despite three decades of active research. Why has it been so hard to tackle sepsis? One obvious reason is the complexity of this disorder as it is thought to be interplay of multiple host immune pathways [3]. The second likely reason is that most of the research has been focused on initiation of inflammatory response when the need is to take into account factors responsible for exaggeration of ongoing inflammation as well as lack of negative regulatory mechanisms/ resolution of inflammation/restoration of homeostasis.

\section{Hyperinflammation: Role of Alarmins}

Sepsis is mainly characterized by an imbalanced, hyperinflammatory response to an infection. As for any pathogenic insult, innate immune components play pivotal role in initiating the proinflammatory response required to combat infection. The key players in this process are host Pattern Recognition Receptors (PRRs) and Pathogen-Associated Molecular Patterns (PAMPs). Three families of host PRRs involved in the recognition of PAMPs are Toll-Like Receptors (TLRS), NOD-Like Receptors (NLRs) and RIG-I like Receptors (RLRs). Regardless of the origin of PAMPs or the localization of PRRs, their interaction almost always results in an inflammatory response that serves as a first line of defense against a wide variety of pathogens. On the flip side, the non-specificity of innate immune response and the antimicrobial mediators produced by the first responder cells such as neutrophils can result in severe damage to by-stander cells at the site of acute inflammation. This causes the release of endogenous host factors in the extracellular milieu. These endogenous molecules are termed alarmins, and typically perform homeostatic functions when contained within cellular compartments. However under pathological conditions, these molecules can be released either passively from dead cells or actively via non-classical secretion pathways. Once in the extracellular milieu, they exhibit immune modulatory properties such as induction of pro-inflammatory cytokines, immune cell chemotaxis and regulation of cell death [4-6].The interaction of Toll-Like Receptors (TLRs) as well as NOD-Like Receptors (NLRs) with pathogen derived PAMPs during sepsis has been studied extensively [3,7]. However, the recognition of self-molecules (alarmins) by signaling receptors and the concomitant inflammatory response is an area of research which is still in its infancy. The role of several alarmins such as High Mobility Group Box 1 (HMGB1) and S100 family of calcium binding proteins has been demonstrated in various inflammatory conditions, as well as in sepsis. However, in a complex immune disorder like sepsis which is interplay of several host immune pathways such as the coagulation system, complement cascade and even the autonomic nervous system, it is likely that several alarmins are involved at the intersections of these pathways. Thus, identification of novel alarmins and their receptors as well as downstream signaling events may aid in understanding this complex disorder and may present additional targets for effective therapeutics. Moreover, depending upon the type of infections detected in septic patients, the identification of these alarmins and alarmin receptors may present opportunities to customize the treatment options in septic patients.

\section{Resolution of Inflammation: Return to Homeostasis}

Once the pathogenic insult has been taken care of, it is imperative that the immune cells are cleared off the mileau, in order to restore the quiescent state of homeostasis. This is achieved by the process of efferocytosis, where the innate immune cells such as neutrophils, once they have completed their task of combating pathogens, start to undergo apoptosis and are eventually phagocytosed by professional phagocytes in the vicinity. Deregulation of this process can result in secondary necrosis of these apoptotic cells causing the release of host alarmins in the extracellular milieu which, we now know, play an important role in exaggeration of an ongoing inflammation. As sepsis is often characterized by massive cell death in systemic organs, it is tempting to speculate that a deregulation of efferocytosis process and turnover of neutrophils contributes to sepsis development. However, studies correlating these two processes with sepsis are surprisingly few. In addition to clearing dead cell carcasses, the process of efferocytosis also modifies the phenotype of phagocytic cells from inflammatory to anti-inflammatory nature resulting in production of host mediators such as TGF-beta and IL-10 that not only shut the inflammatory response but also inhibit further influx of immune cells [8]. All of these events constitute the complex process of resolution. How then, in the context of an inflammatory disorder such as sepsis, or for that matter any inflammatory disease condition, this regulated process of resolution is disrupted and progresses towards an accelerated and sustained condition of chronic inflammation? This is an area of investigation which may hold some key answers to queries regarding the mechanisms of sepsis development and how an otherwise beneficial host response turns to a harmful process of excessive inflammation and overt tissue destruction.

\section{Conclusion}

A lack of understanding of molecular mechanisms tipping the balance between a local, resolving inflammation to a systemic nonresolving inflammatory response has limited our efforts to find effective

*Corresponding author: Jyotika sharma, PhD, Assistant professor, Department of Microbiology and Immunology, UND School of Medicine and Health Sciences, 501 N Columbia Road, Grand Forks, ND 58202-9037, USA, Tel: 701-777-2624; Fax: 701-777-2054; E-mail: jyotika.sharma@med.und.edu

Received September 28, 2012; Accepted September 30, 2012; Published October 02, 2012

Citation: Sharma J (2012) Sepsis: What We Know and What We Don't. J Med Microb Diagn 2:e114. doi:10.4172/2161-0703.1000e114

Copyright: (c) 2012 Sharma J. This is an open-access article distributed under the terms of the Creative Commons Attribution License, which permits unrestricted use, distribution, and reproduction in any medium, provided the original author and source are credited. 
treatment options for sepsis. This knowledge likely holds the key to combating this deadly immune disorder.

\section{References}

1. Angus DC, Linde-Zwirble WT, Lidicker J, Clermont G, Carcillo J, et al. (2001) Epidemiology of severe sepsis in the United States: analysis of incidence, outcome, and associated costs of care. Crit Care Med 29: 1303-1310.

2. Ranieri VM, Thompson BT, Barie PS, Dhainaut JF, Douglas IS, et al. (2012) Drotrecogin alfa (activated) in adults with septic shock. N Engl J Med 366 2055-2064.

3. Rittirsch D, Flierl MA, Ward PA (2008) Harmful molecular mechanisms in sepsis. Nat Rev Immunol 8: 776-787.
4. Bianchi ME (2007) DAMPs, PAMPs and alarmins: all we need to know about danger. J Leukoc Biol 81: 1-5.

5. Manson J, Thiemermann C, Brohi K (2012) Trauma alarmins as activators of damage-induced inflammation. Br J Surg 99: 12-20.

6. Oppenheim JJ, Yang D (2005) Alarmins: chemotactic activators of immune responses. Curr Opin Immunol 17: 359-365.

7. Lamkanfi M, Dixit VM (2011) Modulation of inflammasome pathways by bacterial and viral pathogens. J Immunol 187: 597-602.

8. Kennedy AD, DeLeo FR (2009) Neutrophil apoptosis and the resolution of infection. Immunol Res 43: 25-61. 